\title{
Polyamines modulate the roscovitine-induced cell death switch decision autophagy vs. apoptosis in MCF-7 and MDA-MB-231 breast cancer cells
}

\author{
ELIF DAMLA ARISAN, YUNUS AKKOÇ*, KAAN GENCER AKYÜZ*, EZGI MELEK KERMAN*, \\ PINAR OBAKAN, AJDA ÇOKER-GÜRKAN and NARÇIN PALAVAN ÜNSAL
}

Department of Molecular Biology and Genetics, İstanbul Kültür University, Atakoy Campus, Istanbul 34156, Turkey

Received December 4, 2013; Accepted June 9, 2014

DOI: $10.3892 / \mathrm{mmr} .2015 .3303$

\begin{abstract}
Current clinical strategies against breast cancer mainly involve the use of anti-hormonal agents to decrease estrogen production; however, development of resistance is a major problem. The resistance phenotype depends on the modulation of cell-cycle regulatory proteins, cyclins and cyclin-dependent kinases. Roscovitine, a selective inhibitor of cyclin-dependent kinases, shows high therapeutic potential by causing cell-cycle arrest in various cancer types. Autophagy is a type of cell death characterized by the enzymatic degradation of macromolecules and organelles in double- or multi-membrane autophagic vesicles. This process has important physiological functions, including the degradation of misfolded proteins and organelle turnover. Recently, the switch between autophagy and apoptosis has been proposed to constitute an important regulator of cell death in response to chemotherapeutic drugs. The process is regulated by several proteins, such as the proteins of the Atg family, essential for the initial formation of the autophagosome, and PI3K, important at the early stages of autophagic vesicle formation. Polyamines (PAs) are small aliphatic amines that play major roles in a number of eukaryotic processes, including cell proliferation. The PA levels are regulated by ornithine decarboxylase (ODC), the rate-limiting enzyme in PA biosynthesis. In this study, we aimed to investigate the role of PAs in roscovitine-induced autophagic/apoptotic cell death in estrogen receptor-positive MCF-7 and estrogen receptor-negative MDA-MB-231 breast cancer cells. We show that MDA-MB-231 cells are more resistant to
\end{abstract}

Correspondence to: Dr Elif Damla Arisan, Department of Molecular Biology and Genetics, İstanbul Kültür University, Atakoy Campus, E5 Highway Bakırköy, Istanbul 34156, Turkey

E-mail: d.arisan@iku.edu.tr

*Contributed equally

Key words: breast cancer, polyamines, roscovitine, apoptosis, autophagy roscovitine than MCF-7 cells. This difference was related to the regulation of autophagic key molecules in MDA-MB-231 cells. In addition, we found that exogenous PAs have a role in the cell death decision between roscovitine-induced apoptosis or autophagy in MCF-7 and MDA-MB-231 breast cancer cells.

\section{Introduction}

Breast cancer is one of the most commonly diagnosed cancer types in women, and the second leading cause of cancer-related deaths worldwide (1). Patients with estrogen receptor $\alpha(\mathrm{ER} \alpha)$-positive tumors greatly benefit from existing hormonal therapies. Although anti-estrogens are being used to treat breast cancer $(2,3)$, numerous cases show acquired resistance and irresponsiveness to endocrine therapy, which is a major clinical problem $(3,4)$. Despite the emergence of new promising advances in therapeutics, options to treat hormone-resistant breast tumors are limited, and the mortality rate continues to increase.

It has been suggested, based on a number of findings, that deregulation of cell-cycle components such as cyclin-dependent kinases (CDKs) can contribute to endocrine resistance (5). Therefore, inhibition of CDKs by synthetic, small-molecule drugs has become an attractive therapeutic strategy. Roscovitine is a small, purine-like CDK inhibitor with increased selectivity towards CDK1, CDK2, CDK7 and CDK9 (6-8). Previous studies have shown that roscovitine promotes the accumulation of breast cancer cells at the $\mathrm{G}_{2} / \mathrm{M}$ phase $(9,10)$ and potentiates the antitumor effects of other chemotherapeutic agents, by inducing apoptotic cell death (11). Besides CDKs, the progression of the cell cycle is related to polyamines (PAs), which are amine-derived cationic molecules. Several studies provided evidence for a PA-dependent $G_{0}-G_{1}$ transition and $G_{1}$ phase progression in different cell lines $(12,13)$.

Among PAs, natural putrescine (Put), spermidine (Spd) and spermine (Spm) are required for cell growth and proliferation (14). Intracellular PA levels are tightly regulated in eukaryotes by the activity of the ornithine decarboxylase (ODC), which catalyzes the conversion of ornithine to Put (15). Activation of PA biosynthesis leads to the accumulation of intracellular PAs, which is a critical event in various 
diseases, including breast cancer $(16,17)$. Previous studies have shown that PAs are involved in neoplastic transformation by activating several proto-oncogenes, such as $c$-Myc $(18,19)$.

Autophagy, the process responsible for the degradation of cytoplasmic proteins, macromolecules and damaged or aged organelles, is considered a type of cell death. The most significant sign of autophagy is the appearance of double-membrane enclosed vesicles in the cytoplasm, which engulf portions of the cytoplasm and/or organelles (20-22).

A number of studies have shown that PAs are associated with autophagy via histone acetylation and chromatin remodeling mechanisms. Specifically, Spd was suggested to be a critical 'tuning' molecule in autophagy, through epigenetic alterations (23-25). Spd was shown to inhibit the enzymatic activity of histone acetyl transferase (HAT) and lead to hypoacetylation of histone $\mathrm{H} 3$ (25). For this reason, it is considered that autophagic processes can be activated by the acetylation, by PAs, of autophagic promoter molecules. However, the molecular mechanism involved in drug-induced apoptosis or autophagy related to the regulation of PA biosynthesis has not yet been fully clarified.

In the present study, we aimed to reveal the potential role of PAs in roscovitine-induced apoptosis and/or autophagy in MCF-7 and MDA-MB-231 breast cancer cells.

\section{Materials and methods}

Drugs and antibodies. Roscovitine was purchased from Sigma-Aldrich (St. Louis, MO, USA), was dissolved in dimethyl sulfoxide (DMSO) to make a $10 \mathrm{mM}$ stock solution, and was stored at $-20^{\circ} \mathrm{C}$. Spd, $\mathrm{Spm}$ (each at $10 \mathrm{mM}$ ) and 3-aminoguanidine were purchased from Sigma-Aldrich. 3-Aminoguanidine was used as an amine oxidase blocker in the Spd and Spm treatment experiments.

Antibodies targeting beclin-1 (dilution, 1:1,000), Atg5 $(1: 1,000), \operatorname{Atg} 12(1: 1,000), \mathrm{LC} 3 \mathrm{~A} / \mathrm{B}(1: 1,000), \beta$-actin $(1: 1,000)$, $\beta$-tubulin $(1: 1,000)$, pro-caspase-9 $(1: 1,000)$, cleaved-caspase- 9 $(1: 1,000)$, caspase-7 $(1: 1,000)$ and horseradish peroxidase (HRP)-conjugated secondary IgG $(1: 3,000)$ were purchased from Cell Signaling Technology (Danvers, MA, USA).

Cell cultures. The breast cancer cell lines MCF-7 (HTB 22) and MDA-MB-231 (HTB 26) were purchased from the American Type Culture Collection (ATCC; Manassas, VA, USA). The cells were maintained in Gibco ${ }^{\circledR}$ Dulbecco's modified Eagle's medium (DMEM; Thermo Fisher Scientific, Waltham, MA, USA) supplemented with $10 \%$ fetal bovine serum (Pan-Biotech $\mathrm{GmbH}$, Aidenbach, Germany) and 100 units or $100 \mathrm{mg} / \mathrm{ml}$ penicillin or streptomycin, and were grown in humidified air with $5 \% \mathrm{CO}_{2}$ at $37^{\circ} \mathrm{C}$, in a Heracell ${ }^{\circledR} 150 \mathrm{i}$ incubator (Thermo Fisher Scientific).

Cell viability assay. The effect of roscovitine on cell viability in the presence or absence of PAs was determined by the colorimetric assay 3-(4,5-dimethylthiazol-2-yl)-2,5-diphenyltetrazolium bromide (MTT; Roche Diagnostics, Indianapolis, IN, USA). Cells were plated in 96-well plates at a density of $1 \times 10^{5}$ cells/well, were allowed to attach overnight, and were treated for $24 \mathrm{~h}$ with various concentrations of roscovitine in the presence or absence of PAs. After $24 \mathrm{~h}$ of treatment, $10 \mu \mathrm{l}$ of the MTT reagent $(5 \mathrm{mg} / \mathrm{ml})$ were added to the cell culture medium, and cells were incubated for $4 \mathrm{~h}$. Following medium removal, $200 \mu \mathrm{l}$ of DMSO were added to dissolve the formazan crystals, which are produced by the activated mitochondria. The absorbance of the suspensions was measured at $595 \mathrm{~nm}$ on a microplate reader (Bio-Rad, Hercules, CA, USA).

Fluorescence staining. Cells $\left(5 \times 10^{4}\right)$ were seeded into 12 -well plates, allowed to attach overnight and then treated with appropriate concentrations of drugs for $24 \mathrm{~h}$. In order to assess the mitochondrial membrane potential (MMP), cells were washed once with $1 \mathrm{X}$ phosphate-buffered saline (PBS) and stained with 0,4 mM 3,3'-dihexyloxacarbocyanine iodide ( $\mathrm{DiOC}_{6}$ ). The absorbance of samples (Abs 488/525) was measured on a Fluoroskan Ascent Microplate fluorometer (Thermo Fisher Scientific, Beverly, MA, USA), with excitation and emission settings of 488 and $525 \mathrm{~nm}$, respectively; the Abs 488/525 of the samples was compared to that of the control.

For monodansylcadaverine (MDC) staining, cells $\left(6 \times 10^{4}\right)$ were seeded into 6 -well plates on coverslips, allowed to attach overnight and then treated with the appropriate drug concentrations for $24 \mathrm{~h}$. Cells were washed once with $1 \mathrm{X}$ PBS and stained with $50 \mu \mathrm{M}$ MDC in order to visualize the autophagic vesicles. Next, they were observed under a fluorescence microscope (Olympus, Tokyo, Japan).

Cell death enzyme-linked immunosorbent assay (ELISA) assay. The cytoplasmic histone-associated DNA-fragments (mono- and oligonucleosomes) were measured with the Cell Death Detection ELISA PLUS kit (Roche Diagnostics) according to the manufacturer's instructions. Cells $\left(1 \times 10^{4}\right)$ were seeded into 96-well plates and treated with the desired drug concentrations for $24 \mathrm{~h}$. The cell lysates were placed in a streptavidin-coated microplate. A mixture of anti-histone biotin and anti-DNA peroxidase (POD) was added, and samples were kept at room temperature for $2 \mathrm{~h}$. Following washing of the unbound antibodies, the colorimetric assay was performed with 2,2'-azino-bis(3-ethylbenzthiazoline-6-sulphonic acid as a substrate, and the absorbance was measured at $405 \mathrm{~nm}$.

Immunoblot analysis. Cells were treated with the appropriate concentrations of each drug for $24 \mathrm{~h}$. The MCF-7 and MDA-MB-231 cells were lysed with ProteoJET Mammalian cell lysis reagent (Fermentas, Thermo Fisher Scientific, Waltham, MA, USA) containing total protease inhibitor cocktail (Roche Diagnostics GmbH, Mannheim, Germany).

Following lysis, cell debris was removed by centrifugation for $15 \mathrm{~min}$ at $18,500 \mathrm{xg}$, and protein concentration was determined with the Bradford method (Quick Start ${ }^{\mathrm{TM}}$ Bradford Protein Assay kit; Bio-Rad Laboratories, Hercules, CA, USA). Total protein lysates $(30 \mu \mathrm{g})$ were separated by $15 \%$ SDS-polyacrylamide gel electrophoresis (PAGE) and transferred onto polyvinylidene difluoride membranes (Roche Diagnostics). The membranes were then blocked with $5 \%$ non-fat milk, prepared in a $1 \%$ Tris-buffered saline and Tween-20 (TBST) solution. Following incubation of the membranes with the appropriate primary antibody at $4^{\circ} \mathrm{C}$ overnight, the membranes were washed with TBST. The membranes were then incubated with the appropriate HRP-conjugated secondary antibody overnight at $4{ }^{\circ} \mathrm{C}$, an enhanced chemiluminescence (ECL) reagent (Lumi-Light 
A

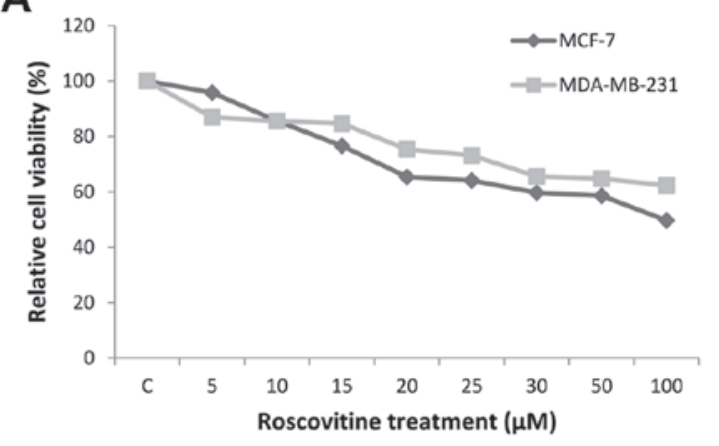

B

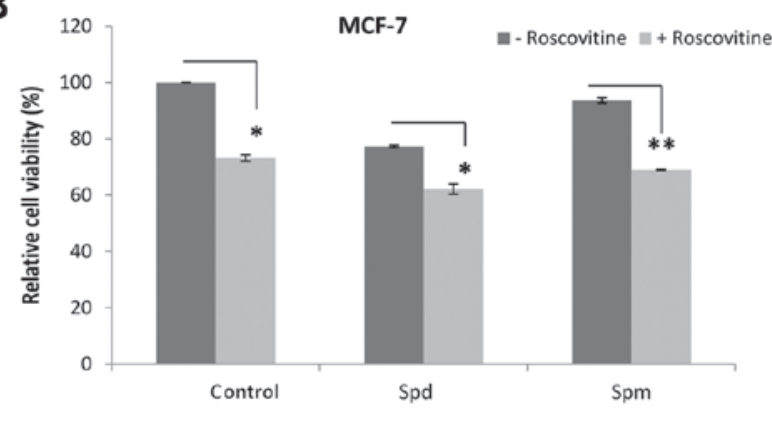

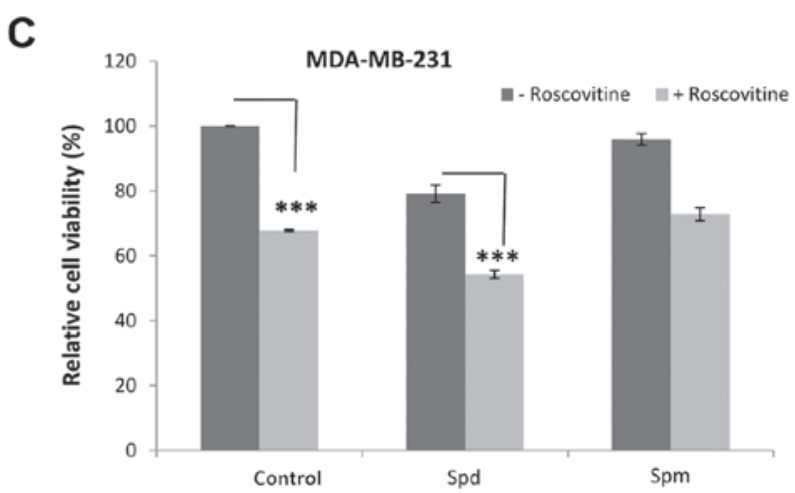

Figure 1. Roscovitine decreases cell viability in a dose-dependent manner in MCF-7 and MDA-MB-231 breast cancer cells. Co-treatment with spermidine (Spd) or spermine (Spm) and roscovitine has distinct effects in each cell line. (A) Cells (1x10 $\left.{ }^{4}\right)$ were seeded into a 96-well plate and treated with roscovitine $(0-100 \mu \mathrm{M})$ for $24 \mathrm{~h}$. The relative cell viability was assessed by the MTT assay. (B and C) The effect of combined treatment with Spd or Spm (each $10 \mu \mathrm{M}$ ) and roscovitine $(20 \mu \mathrm{M})$ for $24 \mathrm{~h}$ on cell viability, as determined by the MTT assay. Columns represent the mean \pm standard deviation from two experiments with 4 replicates. Statistical differences was assessed using an unpaired t-test; ${ }^{*} \mathrm{P}<0.05 ;{ }^{* * *} \mathrm{P}<0.001 ;{ }^{* * * *} \mathrm{P}<0.0001$.

Western Blotting substrate; Roche Diagnostics $\mathrm{GmbH}$ ) was used to visualize the antigens. Finally, the membranes were exposed to Kodak X-ray film (Kodak, Rochester, NY, USA) in a dark room.

Statistical analysis. Differences between samples were statistically evaluated using an Office Excel calculation file (Microsoft, New York, NY, USA). The results from the MTT, cell death ELISA and MMP assay were expressed as mean \pm standard deviation. Student's t-tests were applied toassess the significance of comparisons. Differences were regarded as statistically significant at $\mathrm{P}<0.05$.

\section{Results}

Roscovitine-induced cytotoxicity is altered by polyamine treatment. In order to understand the effect of roscovitine on MCF-7 and MDA-MB-231 breast cancer cells, the MTT cell viability assay was performed following treatment with various concentrations of the drug $(0-100 \mu \mathrm{M})$ for $24 \mathrm{~h}$. The cell viability was decreased by 35 and $25 \%$ following treatment with $20 \mu \mathrm{M}$ roscovitine in MCF-7 and MDA-MB-231 breast cancer cell lines, respectively (Fig. 1A). This concentration was selected for the following experiments.

To evaluate the combined effect of Spd or Spm (each $10 \mu \mathrm{M}$ ) with roscovitine, each cell line was exposed to drugs for $24 \mathrm{~h}$. Although Spd treatment caused moderate cytotoxicity (23\% reduction in cell viability in MCF-7 and $21 \%$ in MDA-MB-231 cells vs. control, respectively), Spm treatment was less effective (7\% in MCF-7 and 4\% in MDA-MB-231 cells) (Fig. 1B and C). Co-treatment with Spd or Spm and roscovitine enhanced the roscovitine-induced cytotoxicity in both breast cancer cell lines. In addition, the promoting effects of Spd on cytotoxicity were significant in both cell lines, particularly in the MDA-MB-231 cells $(\mathrm{P}<0.0002)$.

Roscovitine-induced mitochondria-mediated apoptosis via caspase activation. We determined the apoptotic potential of roscovitine in the presence or absence of $\mathrm{Spd} / \mathrm{Spm}$ in the cells. Although neither Spd nor Spm exerted significant apoptotic effects, roscovitine induced apoptosis by 4 - and 2.5 -fold in MCF-7 and MDA-MB-231 breast cancer cells, respectively, as compared to untreated control cells. Spm alone slightly induced apoptosis in MDA-MB-231 cells by 1.5 -fold compared to control cells. Co-treatment with $\mathrm{Spd}$ or $\mathrm{Spm}$ and roscovitine enhanced the cell viability reduction in both cell lines; it also prevented drug-induced apoptosis by decreasing the DNA fragmentation ratio (Fig. 2).

We performed $\mathrm{DiOC}_{6}$ staining to visualize the MMP loss on a fluorometer and thus, investigate the role of PAs in roscovitine-induced apoptosis. Although roscovitine decreased MMP in both cell lines, co-treatment with Spd did not affect the roscovitine-induced MMP reduction (Fig. 3). By contrast, Spm protected the MCF-7, but not the MDA-MB-231 cells, from roscovitine-induced mitochondria-mediated apoptosis, although theses changes were not significant.

To further investigate drug-induced caspase activation, we determined the level of cleaved fragments of caspase- 9 and -7 by immunoblotting. While caspase- 9 cleavage, which is the initial step for caspase activation in mitochondria-mediated 
A

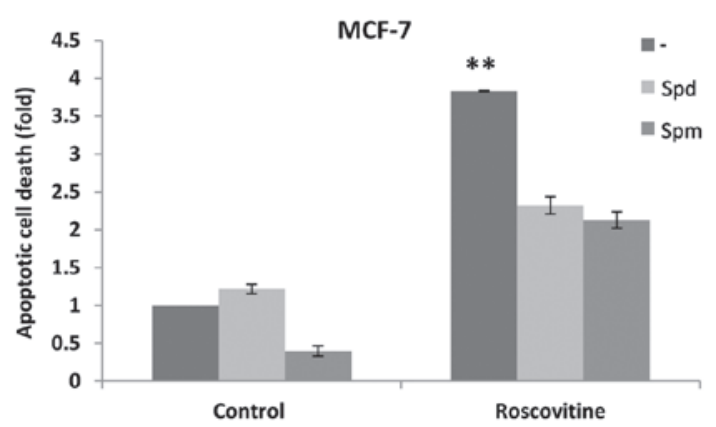

B

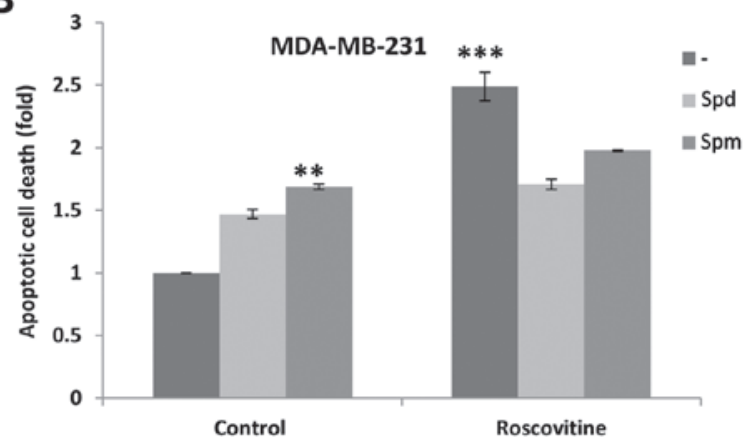

Figure 2. Combined treatment with spermidine (Spd)/spermine (Spm) and roscovitine induces apoptosis in the (A) MCF-7 and (B) MDA-MB-231 breast cancer cell lines. Cells $\left(1 \times 10^{4}\right)$ were seeded into a 96-well plate and treated with roscovitine $(20 \mu \mathrm{M})$ for $24 \mathrm{~h}$. The effect of roscovitine on DNA fragmentation was determined by the Cell Death Detection enzyme-linked immunosorbent assay Plus. Columns represent the mean \pm standard deviation from two experiments with 2 replicates. Statistical difference was analyzed using an unpaired t-test. ${ }^{* *} \mathrm{P}<0.01 ;{ }^{* * *} \mathrm{P}<0.0001$ compared to control samples.
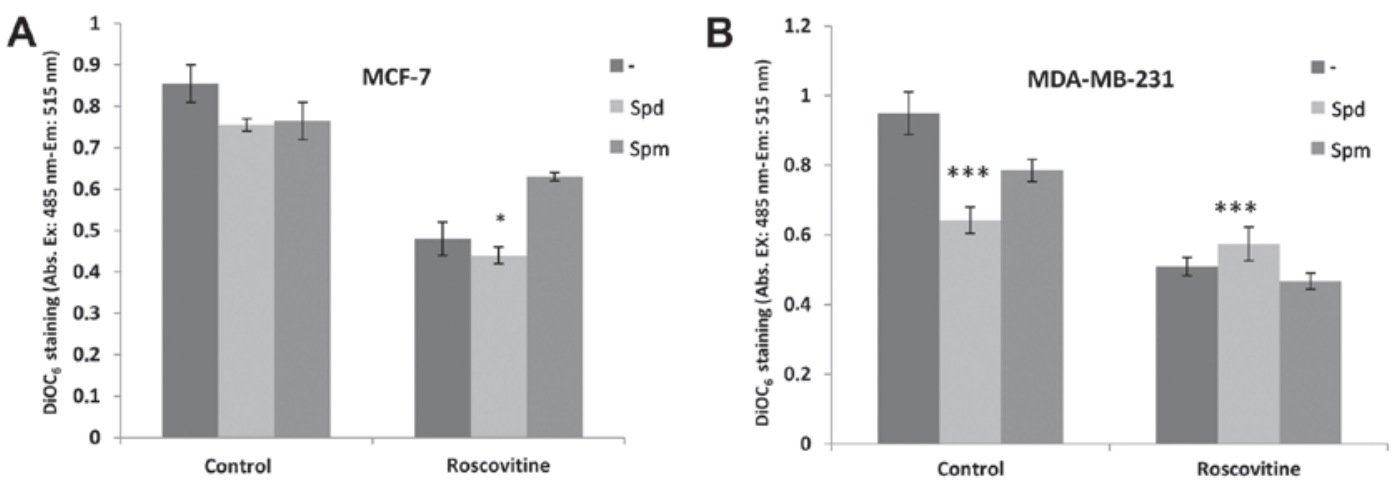

Figure 3. Co-treatment with spermidine (Spd)/spermine (Spm) and roscovitine for $24 \mathrm{~h}$ triggers mitochondria-mediated apoptosis in the (A) MCF-7 and (B) MDA-MB-231 breast cancer cell lines. The effect of roscovitine, alone or in combination with PAs, on the mitochondrial membrane potential was determined by fluorometric analyses following 3,3'-dihexyloxacarbocyanine iodide $\left(\mathrm{DiOC}_{6}\right)$ staining. The y-axes show the absorbance (Abs) ratio at excitation (Ex) wavelength 488 to emissison (Em) wavelength $525 \mathrm{~nm}$. Columns represent the mean \pm standard deviation from two experiments with 4 replicates. Statistical difference was analyzed using an unpaired t-test. ${ }^{*} \mathrm{P}<0.05 ;{ }^{* * * *} \mathrm{P}<0.0001$ compared to control samples.

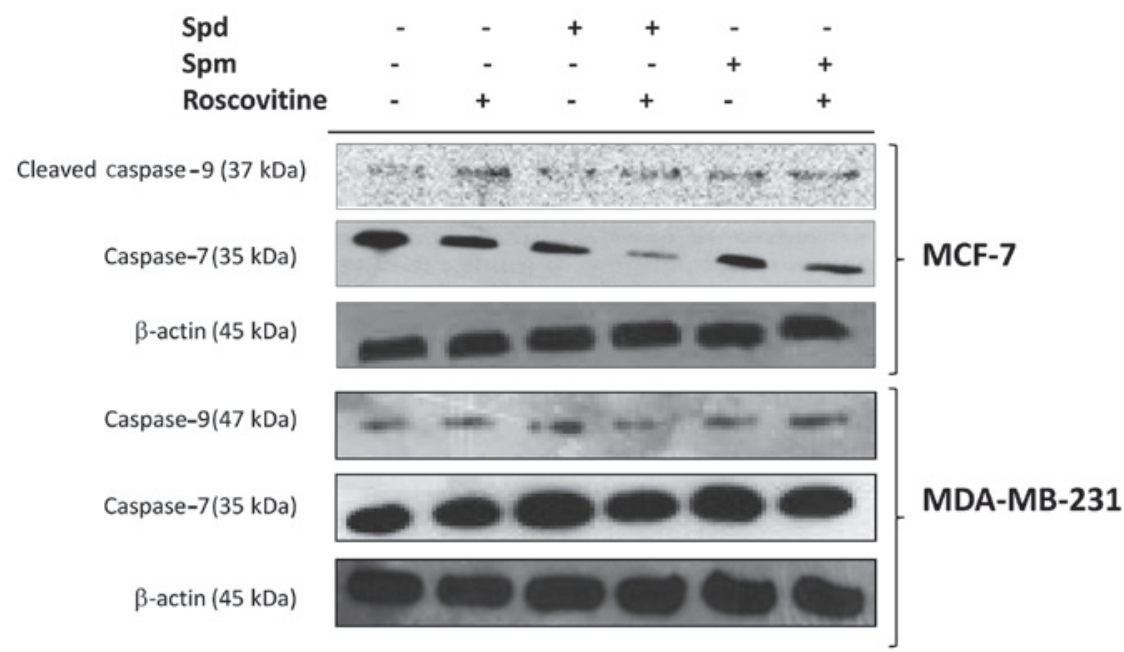

Figure 4. The role of polyamines spermidine (Spd) and spermine (Spm) on roscovitine-induced apoptotic cell death is caspase-dependent in the MCF-7 and MDA-MB-231 breast cancer cell lines, as shown by immunoblotting. Thirty micrograms of total protein lysate were subjected to $15 \%$ sodium dodecyl sulfate-polyacrylamide gel electrophoresis and probed with corresponding antibodies. $\beta$-actin was used as a loading control.

apoptosis, appeared increased, the level of the full-length caspase-7, the executioner caspase for apoptosis, was decreased after roscovitine treatment for $24 \mathrm{~h}$ in breast cancer cells. Although exposure of MCF-7 cells to Spd or Spm for $24 \mathrm{~h}$ did not appear to activate caspase-9, treatment with each of these PAs led to a decrease in the caspase-7 level. In addition, combined treatment with $\mathrm{Spd}$ or $\mathrm{Spm}$ and roscovitine further decreased the expression level of the full-length caspase-7 in 
A

\begin{tabular}{lllllllll}
\multicolumn{8}{c}{ MCF-7 } \\
\multicolumn{8}{c}{ Roscovitine treatment (h) } \\
\hline 0 & 15, & 30, & 1 & 2 & 4 & 8 & 24
\end{tabular}
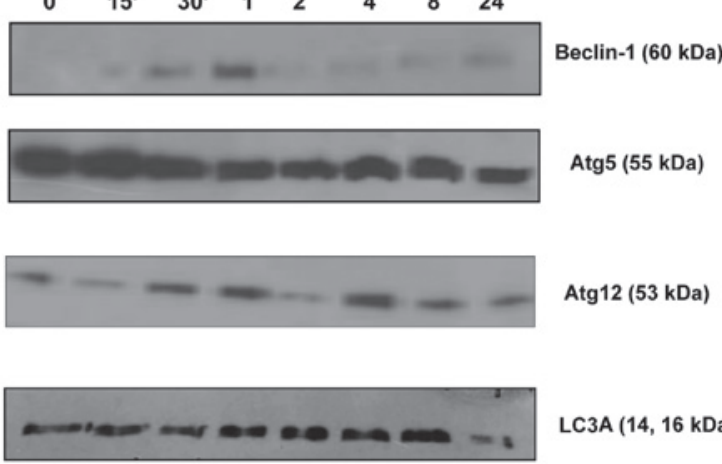

$\operatorname{LC3A}(14,16 \mathrm{kDa})$

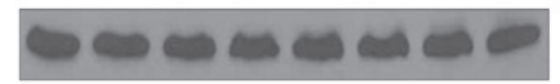

B

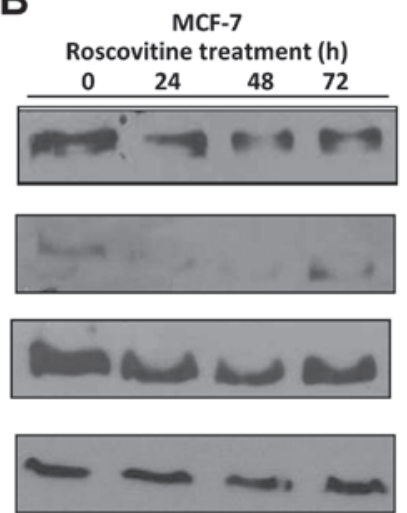

$\beta$-actin (45 kDa)

$\operatorname{Atg} 5$ (55 kDa)

Atg12 (53 kDa)

$\operatorname{LC} 3 \mathrm{~A}(14,16 \mathrm{kDa})$

$\operatorname{Atg} 5$ (55 kDa)

Atg12 (53 kDa)

$\beta$-actin (45 kDa)
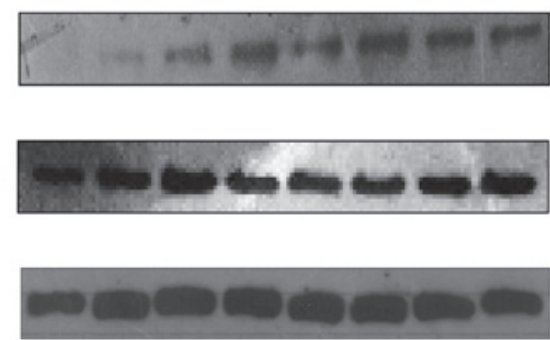

MDA-MB 231

Roscovitine treatment (h)

$\begin{array}{llll}0 & 24 & 48 & 72\end{array}$

MDA-MB-231

Roscovitine treatment (h)

$\begin{array}{llllllll}0 & 15^{\prime} & 30^{\prime} & 1 & 2 & 4 & 8 & 24\end{array}$
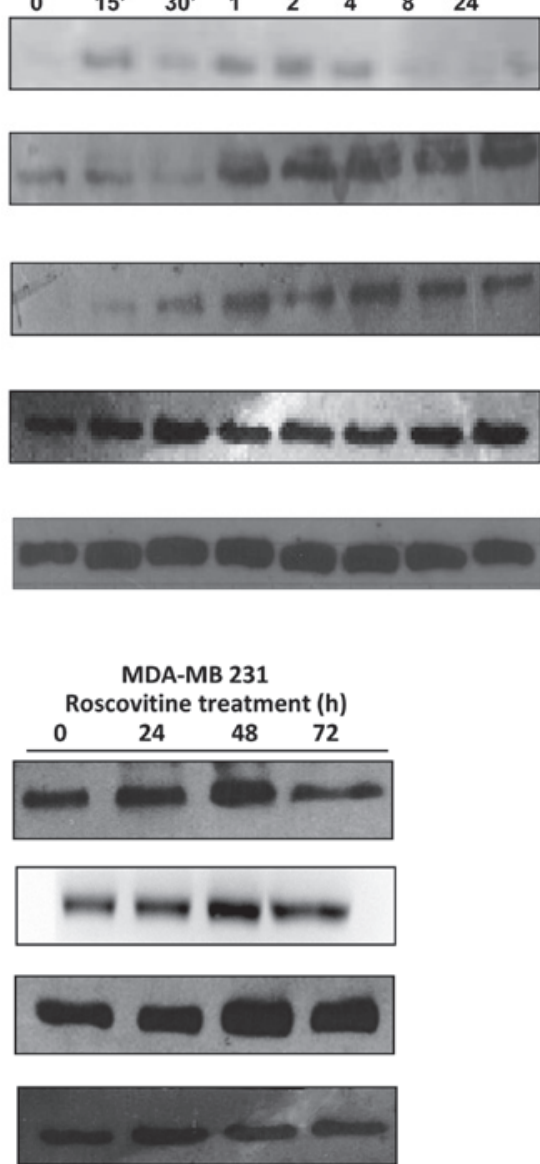

Figure 5. Roscovitine-modulated autophagy in MCF-7 and MDA-MB-231 breast cancer cell lines at different time-points (0-72 h). The expression profiles of (A) beclin-1, Atg5, Atg12 and LC3A/B from 0 to $24 \mathrm{~h}$ ("'” denote minutes) and (B) LC3A/B, Atg5 and Atg12 from 0 to $72 \mathrm{~h}$, as detected by immunoblotting. Thirty micrograms of total protein lysate were subjected to $15 \%$ sodium dodecyl sulfate-polyacrylamide gel electrophoresis and probed with the appropriate antibodies. $\beta$-actin was used as a loading control.

MCF-7 cells. Roscovitine induced the cleavage of caspase-9 and -7 in MDA-MB-231 breast cancer cell lines. In addition, PAs enhanced the roscovitine-induced caspase- 9 and -7 activation by decreasing the level of the full-length fragments of these caspases in MDA-MB-231 cells (Fig. 4).

Roscovitine induces autophagic modulation. In order to evaluate the role of roscovitine on autophagic cell death in the MCF-7 and MDA-MB-231 breast cancer cell lines, we performed immunoblotting assays at different time-points. We examined the expression profile of beclin-1, which is referred to as the initial key molecule for autophagy, following roscovitine treatment within $24 \mathrm{~h}$. Interestingly, while beclin-1 appeared to be time-dependently upregulated from 0 to $1 \mathrm{~h}$ in MCF-7 cells, its expression was stable in MDA-MB-231 cells for up to $4 \mathrm{~h}$.

To indirectly assess the autophagosome complex formation at different time periods of drug treatment, the expression profile of Atg5 and Atg12, which are critical molecules for the elongation of the autophagosomal membrane, were also determined by immunoblotting. The basal expression levels of Atg5 and Atg12 were found to be higher in MCF-7 compared to MDA-MB-231 cells. In general, while roscovitine decreased the expression of Atg5 within $24 \mathrm{~h}$, the Atg5 expression level was increased after $1 \mathrm{~h}$ of drug treatment in the MDA-MB-231 breast cancer cell line (Fig. 5A). Another key marker of autophagosomal formation is LC3A/B, which integrates to double membranes; the level of this protein was increased after $8 \mathrm{~h}$ of roscovitine treatment in MCF-7 cells. However, the expression level of LC3A/B was decreased after $24 \mathrm{~h}$ of drug treatment. When we examined the autophagic effect of roscovitine on LC3A/B expression in MDA-MB-231 cells, we observed a rapid upregulation within $30 \mathrm{~min}$ and an overall higher basal level compared to MCF-7 cells. In addition, LC3A/B expression was higher in MDA-MB-231 cells after $24 \mathrm{~h}$ of drug treatment compared to MCF-7 cells. When the MCF-7 cells were treated with roscovitine for $72 \mathrm{~h}$, the expression of the autophagic key markers LC3A/B, Atg5 and Atg12 was decreased from the first $24 \mathrm{~h}$. However, the protein levels of these markers appeared increased again after $72 \mathrm{~h}$ of drug treatment in MCF-7 cells. By contrast, the expression levels of LC3A/B, Atg5 and Atg12 appeared increased after $48 \mathrm{~h}$ of drug treatment in the MDA-MB-231 cell line (Fig. 5B). Based on these results, we conclude that MCF-7 cells are more sensitive to roscovitine-induced autophagy than MDA-MB-231 cells. 
A
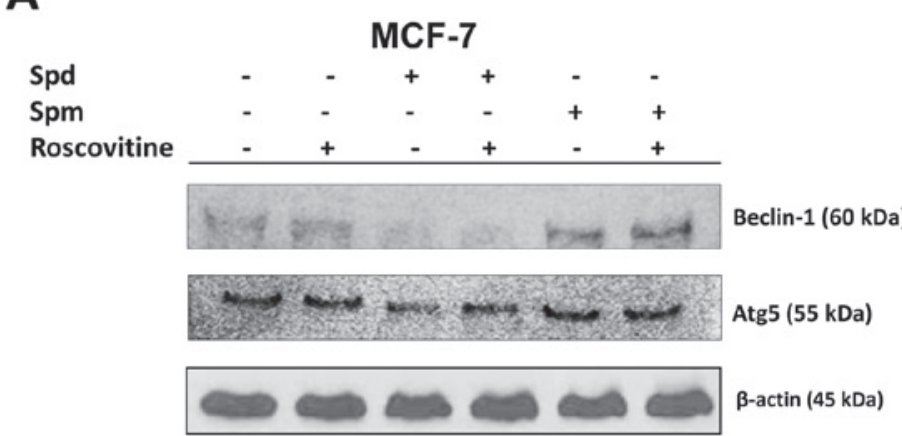

MDA-MB-231

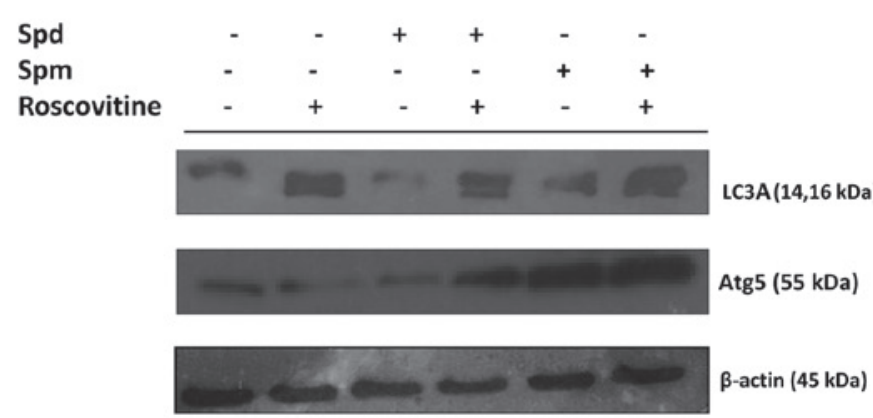

B

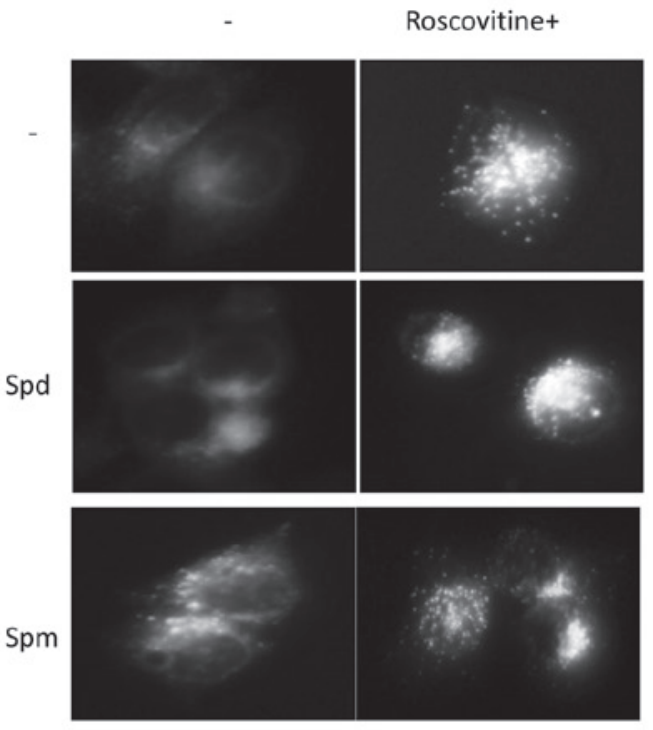

Figure 6. The role of polyamines on roscovitine-induced autophagy in the MCF-7 and MDA-MB-231 breast cancer cell lines. (A) Beclin-1, Atg5 and LC3A/B expression profiles were determined by immunoblotting following $24 \mathrm{~h}$ of spermidine (Spd) or spermine (Spm) co-treatment with roscovitine. Thirty micrograms of total protein lysate were subjected to $15 \%$ sodium dodecyl sulfate-polyacrylamide gelelectrophoresis and then probed with the appropriate antibodies. $\beta$-actin was used as a loading control. (B) The role of polyamines on roscovitine-induced autophagy was determined after $24 \mathrm{~h}$ of treatment with roscovitine alone or in combination with PAs in MCF-7 cells. Following monodansylcadaverine (MDC) staining, fluorescence microscopy allowed to visualize the formation of autophagic vacuoles.

Polyamines modulate roscovitine induced autophagy. In order to further explore the role of polyamines in drug-induced autophagy, cells were treated with roscovitine in the presence of Spd or Spm for $24 \mathrm{~h}$. Spd was not an autophagy inducer but Spm was a good candidate to induce autophagy by upregulating beclin-1 and Atg5 in MCF-7 and MDA-MB-231 cells (Fig. 6A). Moreover, cleaved fragments of LC3A/B were observed following Spm treatment in MDA-MB-231 cells. After treatment with Spd alone, beclin-1 and Atg5 expression levels decreased. By contrast, after treatment with Spm alone, the beclin-1 and Atg5 expression levels increased. Co-treatment with roscovitine and $\mathrm{Spm}$ showed opposite effects on autophagic marker expression in the two breast cancer cell lines compared to co-treatment with roscovitine and Spd (Fig. 6A). These results were confirmed in MCF-7 cells by MDC staining, which allows to detect the autophagic vacuoles (Fig. 6B).

\section{Discussion}

The majority of malignancies are associated with the loss of functional cell-cycle control, which results in impaired apoptosis and unlimited growth. An emerging anticancer approach is to control the aberrant cell cycle machinery by evaluating key molecules for drug design. As shown in previous studies, CDK inhibitors exert their apoptotic effect by causing cell-cycle arrest $(10,26,27)$. Roscovitine is a promising CDK inhibitor with high apoptotic potential in malignant cells. It competitively binds to the ATP binding site of CDKs and prevents cyclin-CDK complex formation (28-31). Furthermore, roscovitine is the first orally bioavailable CDK inhibitor in clinical trials for B-cell malignancies and lung cancer $(31,32)$. A previous study indicated that roscovitine induces apoptosis in breast cancer cells by causing cell-cycle arrest at the $G_{2} / M$ phase (9). PAs are key regulators of cellular processes such as transcription, translation and proliferation (33). PA metabolic enzymes have been proposed as targets for antineoplastic therapy in breast cancer, since their high intracellular level was found associated with rapid cel-cycle turnover in these cells compared to healthy breast tissue cells (14,34-37). In the present study, we demonstrated that roscovitine decreases cell viability in a dose-dependent manner in the MCF-7 and MDA-MB-231 breast cancer cell lines (Fig. 1A). We also determined that the combination of Spd or Spm with roscovitine can enhance drug-induced cytotoxicity in both breast cancer cell lines (Fig. 1B and C). MCF-7 and MDA-MB-231 cells have a different expression status for $\mathrm{ER} \alpha$, which regulates the transcription of genes such as $C D K 2$, a target of roscovitine. CDK2 has been also shown to enhance the ligand-independent ER $\alpha$ activation (38-40), which indicates that this protein can play a critical role in the responsiveness against the hormone ablation therapy $(5,41,42)$. Similar to previous findings $(9,10)$, roscovitine inhibited the proliferation rates to different degrees in ER $\alpha$-positive and -negative breast cancer cell lines in our study.

Exposure of cancer cells to PAs may affect the modulation of cell responses to drug treatment in a cell-dependent manner. While Spd treatment protected Erhlich ascite tumor cells 
against apoptosis triggered by acetoxychavicol acetate (43), Spm was shown to synergistically act with bovine serum oxidase in docetaxel-induced apoptosis in MCF-7 cells (44). According to a previous study by our group, roscovitine-induced apoptotic cell death may be altered when PA biosynthesis is inhibited in HCT116 colon carcinoma cells (45).

Although increased accumulation of intracellular PAs is associated with disease progression and rapid cell-cycle turnover, due to high PA catabolic activity, Spm may induce apoptosis by activating cellular caspases (46-48).

Cell death in vertebrates mostly proceeds via the mitochondrial pathway in a caspase-dependent manner $(49,50)$. CDK inhibitors were shown to exert their apoptotic effect through inducing MMP loss and activating caspases in cancer cells $(51,52)$. Similar to these observations, we determined that exposure of cells to roscovitine for $24 \mathrm{~h}$ induces the modulation of MMP in the MCF-7 and MDA-MB-231 breast cancer cell lines. However, combined treatment of Spd or Spm with roscovitine caused different effects in both cell lines. Spm protected cells against roscovitine-induced mitochondria-mediated apoptosis in MCF-7, but not in MDA-MB-231 breast cancer cells (Fig. 3). According to the results of the present study, there was a difference in the two breast cancer cell lines treated with roscovitine and PAs, with regards to cell death response. The MCF-7 and MDA-MB-231 cell lines have different genetic backgrounds, particularly with regards to ER status, which is associated with cellular growth and the fate of cells. Therefore, it may be hypothesized that the difference in cell death response between these cell lines is due to key targets within the ER. Furthermore, hormone signaling may promote different cell signaling pathways to induce either apoptosis or autophagy $(2,16)$. In addition, PAs also have a role in cell growth, and the treatment of the cells with PAs resulted in an altered cell response to roscovitine treatment. Therefore, MCF-7 and MDA-MB-231 cells may act differently upon drug exposure. In association with these data, we showed that in the MCF-7 breast cancer cell line, roscovitine treatment for $24 \mathrm{~h}$ results in the cleavage of pro-caspase- 9 and -7 , which is referred to in the literature as the initial eevent during induction of apoptosis. Upon treatment with roscovitine, additional Spm exposure affected the activation of both caspases in the MCF-7 and MDA-MB-231 breast cancer cell lines. However, treatment with roscovitine only did not exert the same effect on the MDA-MB-231 cells (Fig. 4).

In the second part of the present study, we investigated the role of the CDK inhibitor on autophagy and the potential role of PAs on autophagic regulation in MCF-7 and MDA-MB-231 breast cancer cell lines. The therapeutic efficiencies of drug candidates for cancer treatment were investigated in recent studies by examination of their potential to activate both apoptosis and autophagy, and by studying their interactions $(53,54)$. Therefore, elucidation of the molecular mechanism common to apoptosis and autophagy, as well as of the crosstalk between these two processes is of high importance. Inhibition of autophagy has been shown to enhance the induction of apoptosis $(55,56)$. Under cellular stress conditions, such as in the presence of DNA-damaging agents, autophagy is inhibited and the intrinsic pathway of apoptosis is triggered in MCF-7 cells, but the induction of autophagy can delay apoptosis (57).
In association with these findings, we found that 24-h treatment with roscovitine modulates the mechanism underlying autophagy in MDA-MB-231, but not in MCF-7 cells (Fig. 5A). Longer exposure of both cell lines to roscovitine confirmed that the autophagic process is more prominent in MDA-MB-231 cells compared to MCF-7 cells. Therefore, we conclude that MCF-7 cells are more sensitive to roscovitine-induced autophagy than MDA-MB-231 cells (Fig. 5B).

In general, autophagy delays cell death and prolongs the lifespan in various experimental aging models (58-60). Recent studies showed that PAs, and in particular Spd, induce autophagy and cause increased lifespan. For instance, naphthalimide-PA conjugates trigerred autophagy by modulating the mTOR signaling cascade. Exposure of HepG2 cells to the naphthalimide-PA conjugates induced autophagic vesicle formation $(61,62)$. In a similar way, Spd treatment can induce LC3 formation in HeLa cells (63). Therefore, Spd-induced autophagy may be therapeutically useful for cancer treatment. Indeed, increased levels of highly and positively-charged PAs have been found to correlate with chromatin condensation, and to modulate HAT and HDAC activities in murine skin tumors $(24,25)$. However, in yeast, Spd treatment has been shown to trigger the global hypoacetylation of histone $\mathrm{H} 3$ and selectively acetylate the promoter region of the atg7 gene, which led to the upregulation of autophagic genes $(23,63)$. According to our findings, Spm may be proposed as an autophagic agent in MCF-7 and MDA-MB-231 cells (Fig. 6).

Therefore, we conclude that roscovitine is a mediator of apoptosis in the ER $\alpha^{+} \mathrm{MCF}-7$ breast cancer cells, and that apoptosis is delayed by the induction of autophagy in ER $\alpha$ MDA-MB-231 cells. In addition, PAs play critical roles in roscovitine-induced autophagy in a cell type-dependent manner.

\section{References}

1. Jemal A, Siegel R, Ward E, Hao Y, Xu J and Thun MJ: Cancer statistics, 2009. CA Cancer J Clin 59: 225-249, 2009.

2. Lerner LJ and Jordan VC: Development of antiestrogens and their use in breast cancer: eighth Cain memorial award lecture. Cancer Res 50: 4177-4189, 1990.

3. Jaiyesimi IA, Buzdar AU, Decker DA and Hortobagyi GN: Use of tamoxifen for breast cancer: twenty-eight years later. J Clin Oncol 13: 513-529, 1995.

4. Buzdar AU and Hortobagyi G: Update on endocrine therapy for breast cancer. Clin Cancer Res 4: 527-534, 1998.

5. Nair BC and Vadlamudi RK: Regulation of hormonal therapy resistance by cell cycle machinery. Gene Ther Mol Biol 12: 395 , 2008.

6. Al-Minawi AZ, Saleh-Gohari $\mathrm{N}$ and Helleday T: The $\mathrm{ERCC1/XPF}$ endonuclease is required for efficient single-strand annealing and gene conversion in mammalian cells. Nucleic Acids Res 36: 1-9, 2008.

7. Hunt T: You never know: Cdk inhibitors as anti-cancer drugs. Cell Cycle 7: 3789-3790, 2008.

8. Aldoss IT, Tashi T and Ganti AK: Seliciclib in malignancies. Expert Opin Investig Drugs 18: 1957-1965, 2009.

9. Wojciechowski J, Horky M, Gueorguieva $M$ and Wesierska-Gadek J: Rapid onset of nucleolar disintegration preceding cell cycle arrest in roscovitine-induced apoptosis of human MCF-7 breast cancer cells. Int J Cancer 106: 486-495, 2003.

10. Wesierska-Gadek J, Gueorguieva $M$ and Horky $M$ : Roscovitine-induced up-regulation of p53AIP1 protein precedes the onset of apoptosis in human MCF-7 breast cancer cells. Mol Cancer Ther 4: 113-124, 2005. 
11. Appleyard MV, O'Neill MA, Murray KE, et al: Seliciclib (CYC202, R-roscovitine) enhances the antitumor effect of doxorubicin in vivo in a breast cancer xenograft model. Int J Cancer 124: 465-472, 2009.

12. Charollais RH and Mester J: Resumption of cell cycle in BALB/c-3T3 fibroblasts arrested by polyamine depletion: relation with 'competence' gene expression. J Cell Physiol 137: 559-564, 1988

13. Harada JJ and Morris DR: Cell cycle parameters of Chinese hamster ovary cells during exponential, polyamine-limited growth. Mol Cell Biol 1: 594-599, 1981

14. Pegg AE: Polyamine metabolism and its importance in neoplastic growth and a target for chemotherapy. Cancer Res 48: 759-774, 1988.

15. Deng W, Jiang X, Mei Y, et al: Role of ornithine decarboxylase in breast cancer. Acta Biochim Biophys Sin (Shanghai) 40: 235-243, 2008.

16. Hoggard N and Green CD: Polyamines and growth regulation of cultured human breast cancer cells by 17 beta-oestradiol. Mol Cell Endocrinol 46: 71-78, 1986.

17. Manni A: Polyamine involvement in breast cancer phenotype. In Vivo 16: 493-500, 2002.

18. Bello-Fernandez C, Packham G and Cleveland JL: The ornithine decarboxylase gene is a transcriptional target of c-Myc. Proc Natl Acad Sci USA 90: 7804-7808, 1993

19. Celano P, Baylin SB, Giardiello FM, Nelkin BD and Casero RA Jr: Effect of polyamine depletion on c-myc expression in human colon carcinoma cells. J Biol Chem 263: 5491-5494, 1988.

20. Kondo Y, Kanzawa T, Sawaya R and Kondo S: The role of autophagy in cancer development and response to therapy. Nat Rev Cancer 5: 726-734, 2005.

21. Gozuacik D and Kimchi A: Autophagy and cell death. Curr Top Dev Biol 78: 217-245, 2007.

22. Maiuri MC, Zalckvar E, Kimchi A and Kroemer G: Self-eating and self-killing: crosstalk between autophagy and apoptosis. Nat Rev Mol Cell Biol 8: 741-752, 2007.

23. Madeo F, Eisenberg T, Büttner S, Ruckenstuhl C and Kroemer G: Spermidine: a novel autophagy inducer and longevity elixir. Autophagy 6: 160-162, 2010.

24. Hobbs CA and Gilmour SK: High levels of intracellular polyamines promote histone acetyltransferase activity resulting in chromatin hyperacetylation. J Cell Biochem 77: 345-360, 2000.

25. Hobbs CA, Paul BA and Gilmour SK: Deregulation of polyamine biosynthesis alters intrinsic histone acetyltransferase and deacetylase activities in murine skin and tumors. Cancer Res 62 67-74, 2002.

26. Wesierska-Gadek J, Wandl S, Kramer MP, Pickem C, Krystof V and Hajek SB: Roscovitine up-regulates p53 protein and induces apoptosis in human HeLaS(3) cervix carcinoma cells. J Cell Biochem 105: 1161-1171, 2008.

27. Wesierska-Gadek J, Gueorguieva M, Wojciechowski J and Horky M: Cell cycle arrest induced in human breast cancer cells by cyclin-dependent kinase inhibitors: a comparison of the effects exerted by roscovitine and olomoucine. Pol J Pharmacol 56 635-641, 2004

28. Meijer L, Borgne A, Mulner O, et al: Biochemical and cellular effects of roscovitine, a potent and selective inhibitor of the cyclin-dependent kinases cdc2, cdk2 and cdk5. Eur J Biochem 243: 527-536, 1997.

29. Fischer PM and Gianella-Borradori A: CDK inhibitors in clinical development for the treatment of cancer. Expert Opin Investig Drugs 12: 955-970, 2003

30. Hahntow IN, Schneller F, Oelsner M, et al: Cyclin-dependent kinase inhibitor Roscovitine induces apoptosis in chronic lymphocytic leukemia cells. Leukemia 18: 747-755, 2004.

31. Decker T, Hipp S, Hahntow I, Schneller F and Peschel C: Expression of cyclin $\mathrm{E}$ in resting and activated $\mathrm{B}$-chronic lymphocytic leukaemia cells: cyclin E/cdk2 as a potential therapeutic target. Br J Haematol 125: 141-148, 2004.

32. Benson C, White J, De Bono J, et al: A phase I trial of the selective oral cyclin-dependent kinase inhibitor seliciclib (CYC202; R-Roscovitine), administered twice daily for 7 days every 21 days. Br J Cancer 96: 29-37, 2007.

33. Zaletok S, Alexandrova N, Berdynskykh N, et al: Role of polyamines in the function of nuclear transcription factor NF-kappaB in breast cancer cells. Exp Oncol 26: 221-225, 2004.

34. Wang Y and Casero RA Jr: Mammalian polyamine catabolism: a therapeutic target, a pathological problem, or both? J Biochem 139: 17-25, 2006
35. Persson L and Rosengren E: Increased formation of N1-acetylspermidine in human breast cancer. Cancer Lett 45: 83-86, 1989

36. Cañizares F, Salinas J, de las Heras M, et al: Prognostic value of ornithine decarboxylase and polyamines in human breast cancer: correlation with clinicopathologic parameters. Clin Cancer Res 5: 2035-2041, 1999.

37. Casero RA Jr and Marton LJ: Targeting polyamine metabolism and function in cancer and other hyperproliferative diseases. Nat Rev Drug Discov 6: 373-390, 2007.

38. Ali S and Coombes RC: Endocrine-responsive breast cancer and strategies for combating resistance. Nat Rev Cancer 2: 101-112, 2002.

39. Loi S, Haibe-Kains B, Desmedt C, et al: Definition of clinically distinct molecular subtypes in estrogen receptor-positive breast carcinomas through genomic grade. J Clin Oncol 25: 1239-1246, 2007.

40. Sutherland RL and Musgrove EA: CDK inhibitors as potential breast cancer therapeutics: new evidence for enhanced efficacy in $\mathrm{ER}^{+}$disease. Breast Cancer Res 11: 112, 2009.

41. Rogatsky I, Trowbridge JM and Garabedian MJ: Potentiation of human estrogen receptor alpha transcriptional activation through phosphorylation of serines 104 and 106 by the cyclin A-CDK2 complex. J Biol Chem 274: 22296-22302, 1999.

42. Trowbridge JM, Rogatsky I and Garabedian MJ: Regulation of estrogen receptor transcriptional enhancement by the cyclin $\mathrm{A} / \mathrm{Cdk} 2$ complex. Proc Natl Acad Sci USA 94: 10132-10137, 1997.

43. Moffatt J, Hashimoto M, Kojima A, et al: Apoptosis induced by 1 -acetoxychavicol acetate in Ehrlich ascites tumor cells is associated with modulation of polyamine metabolism and caspase- 3 activation. Carcinogenesis 21: 2151-2157, 2000.

44. Marra M, Lombardi A, Agostinelli E, et al: Bovine serum amine oxidase and spm potentiate docetaxel and interferon-alpha effects in inducing apoptosis on human cancer cells through the generation of oxidative stress. Biochim Biophys Acta 1783: 2269-2278, 2008.

45. Arisan ED, Coker A and Palavan-Ünsal N: Polyamine depletion enhances the roscovitine-induced apoptosis through the activation of mitochondria in HCT116 colon carcinoma cells. Amino Acids 42: 655-665, 2012.

46. Xie X, Tome ME and Gerner EW: Loss of intracellular putrescine pool-size regulation induces apoptosis. Exp Cell Res 230: 386-392, 1997.

47. Stefanelli C, Bonavita F, Stanic I, et al: Spermine causes caspase activation in leukaemia cells. FEBS Lett 437: 233-236, 1998.

48. Stefanelli C, Stanic I, Zini M, et al: Polyamines directly induce release of cytochrome $\mathrm{c}$ from heart mitochondria. Biochem J 347: $875-880,2000$

49. Ouyang L, Shi Z, Zhao S, et al: Programmed cell death pathways in cancer: a review of apoptosis, autophagy and programmed necrosis. Cell Prolif 45: 487-498, 2012.

50. Green DR and Kroemer G: The pathophysiology of mitochondrial cell death. Science 305: 626-629, 2004

51. Yenugonda VM, Deb TB, Grindrod SC, et al: Fluorescent cyclin-dependent kinase inhibitors block the proliferation of human breast cancer cells. Bioorg Med Chem 19: 2714-2725, 2011.

52. Ringer L, Sirajuddin P, Yenugonda VM, et al: VMY-1-103, a dansylated analog of purvalanol $\mathrm{B}$, induces caspase-3-dependent apoptosis in LNCaP prostate cancer cells. Cancer Biol Ther 10: 320-325, 2010.

53. Liu B, Wu JM, Li J, et al: Polygonatum cyrtonema lectin induces murine fibrosarcoma L929 cell apoptosis and autophagy via blocking Ras-Raf and PI3K-Akt signaling pathways. Biochimie 92: 1934-1938, 2010.

54. Cheng Y, Qiu F, Huang J, Tashiro S, Onodera S and Ikejima T: Apoptosis-suppressing and autophagy-promoting effects of calpain on oridonin-induced L929 cell death. Arch Biochem Biophys 475: 148-155, 2008.

55. Lambert LA, Qiao N, Hunt KK, et al: Autophagy: a novel mechanism of synergistic cytotoxicity between doxorubicin and roscovitine in a sarcoma model. Cancer Res 68: 7966-7974, 2008.

56. Amaravadi RK, Yu D, Lum JJ, et al: Autophagy inhibition enhances therapy-induced apoptosis in a Myc-induced model of lymphoma. J Clin Invest 117: 326-336, 2007.

57. Boya P, González-Polo RA, Casares N, et al: Inhibition of macroautophagy triggers apoptosis. Mol Cell Biol 25: 1025-1040, 2005.

58. Abedin MJ, Wang D, McDonnell MA, Lehmann U and Kelekar A: Autophagy delays apoptotic death in breast cancer cells following DNA damage. Cell Death Differ 14: 500-510, 2007. 
59. Jia K and Levine B: Autophagy is required for dietary restriction-mediated life span extension in C. elegans. Autophagy 3: 597-599, 2007.

60. Meléndez A, Tallóczy Z, Seaman M, Eskelinen EL, Hall DH and Levine B: Autophagy genes are essential for dauer development and life-span extension in C. elegans. Science 301: 1387-1391, 2003.

61. Tavernarakis N, Pasparaki A, Tasdemir E, Maiuri MC and Kroemer G: The effects of p53 on whole organism longevity are mediated by autophagy. Autophagy 4: 870-873, 2008.
62. Tian ZY, Xie SQ, Mei ZH, Zhao J, Gao WY and Wang CJ: Conjugation of substituted naphthalimides to polyamines as cytotoxic agents targeting the Akt/mTOR signal pathway. Org Biomol Chem 7: 4651-4660, 2009.

63. Eisenberg T, Knauer H, Schauer A, et al: Induction of autophagy by spermidine promotes longevity. Nat Cell Biol 11: 1305-1314, 2009. 